\title{
Masking Disrupts Reentrant Processing in Human Visual Cortex
}

\author{
J. J. Fahrenfort ${ }^{1}$, H. S. Scholte ${ }^{1}$, and V. A. F. Lamme $^{1,2}$
}

\begin{abstract}
In masking, a stimulus is rendered invisible through the presentation of a second stimulus shortly after the first. Over the years, authors have typically explained masking by postulating some early disruption process. In these feedforwardtype explanations, the mask somehow "catches up" with the target stimulus, disrupting its processing either through lateral or interchannel inhibition. However, studies from recent years indicate that visual perception-and most notably visual awareness itself-may depend strongly on cortico-cortical feedback connections from higher to lower visual areas. This has led some researchers to propose that masking derives its effectiveness from selectively interrupting these reentrant processes. In this experiment, we used electroencephalogram measurements to determine what happens in the human visual
\end{abstract}

\section{INTRODUCTION}

In backward masking, a target stimulus is rendered less perceptible or even invisible through the presentation of a second stimulus, the mask. By now, a respectable number of studies have investigated the neural basis of visual masking, both in its own right and as a corollary to studying visual perception and awareness. In explaining a variety of masking phenomena, some have emphasized low-level lateral inhibitory mechanisms (Macknik \& Livingstone, 1998; Francis, 1997), whereas others have postulated target disruption at the precategorical level through interchannel inhibition (Breitmeyer, Ro, \& Ogmen, 2004; Ogmen, Breitmeyer, \& Melvin, 2003).

However, early studies, as well as more recent imaging studies on masked priming, show that processing of masked targets may continue well beyond the precategorical level (Naccache et al., 2005; Dehaene et al., 1998, 2001; Eimer \& Schlaghecken, 1998; Greenwald, Klinger, \& Liu, 1989). These results suggest that masking does not disrupt target processing at an early stage. This is in line with various neurophysiological reports in which early transient responses to undetected masked stimuli

\footnotetext{
${ }^{1}$ University of Amsterdam, ${ }^{2}$ Netherlands Institute for Neuroscience, Amsterdam
}

cortex during detection of a texture-defined square under nonmasked (seen) and masked (unseen) conditions. Electroencephalogram derivatives that are typically associated with reentrant processing turn out to be absent in the masked condition. Moreover, extrastriate visual areas are still activated early on by both seen and unseen stimuli, as shown by scalp surface Laplacian current source-density maps. This conclusively shows that feedforward processing is preserved, even when subject performance is at chance as determined by objective measures. From these results, we conclude that masking derives its effectiveness, at least partly, from disrupting reentrant processing, thereby interfering with the neural mechanisms of figure-ground segmentation and visual awareness itself.

were measured in high visual areas such as the inferotemporal cortex, and even in visual neurons of the frontal eye field (Thompson \& Schall, 1999; Kovacs, Vogels, \& Orban, 1995; Rolls \& Tovee, 1994).

This has resulted in a number of researchers proposing that masking disrupts reentrant cortical signals, while largely leaving signals related to feedforward processing intact (Ro, Breitmeyer, Burton, Singhal, \& Lane, 2003; Lamme, Zipser, \& Spekreijse, 2002; Di Lollo, Enns, \& Rensink, 2000; Bridgeman, 1980). These proposals build on the notion that besides feedforward connections from low to higher visual areas, roughly equal numbers of recurrent or reentrant connections exist going back from higher to lower visual areas (Felleman \& Van Essen, 1991). Such feedback connections have been suggested to play an integral role in a range of processes, such as figure-ground segmentation, attention, awareness, predictive coding, as well as perception of visual detail (Spratling \& Johnson, 2004; Hochstein \& Ahissar, 2002; Kastner \& Ungerleider, 2000; Lamme \& Roelfsema, 2000; Rao \& Ballard, 1999; Luck, Chelazzi, Hillyard, \& Desimone, 1997; Lamme, 1995). It is not unlikely that masking derives its effectiveness (at least in part) from disrupting reentrant signals, while leaving feedforward signals intact, thereby interfering with mechanisms thought to mediate visual awareness itself (Lamme \& Roelfsema, 2000). 
However, many masking studies do not show a relation between reentrant processing and masking. This may be caused by the fact that many such studies make use of luminance or color contrast targets and metacontrast masks. In such metacontrast masking paradigms, the mask tightly fits the preceding target, and the common contour has a contrast of opposite polarity (Becker \& Anstis, 2004). It is quite likely that low-level lateral inhibitory mechanisms come into play when processing these types of stimuli, at least at sufficiently short stimulus onset asynchronies (Macknik \& MartinezConde, 2004; Macknik \& Livingstone, 1998).

Contrast sensitivity is fundamental to vision and contrast is greatly enhanced by lateral inhibition at many levels of visual processing, starting in the retina (Hartline, 1949). Therefore, the use of (meta)contrast stimuli without appropriately controlling for the influence of contrast on the signal exaggerates the influence of low-level lateral inhibition, while obscuring the influence of other higherlevel processes, such as reentrant processing. The stimuli used in this experiment-in combination with a subtraction method (detailed below) - eliminate the influence of luminance contrast on the signal, while at the same time controlling for other low-level influences.

Aside from the contrast issue, visibility of masked targets is often assessed using an identification task. However, a detection task is more fundamentally connected to the notion of visual awareness, as many studies demonstrate that residual identification in the absence of awareness exists. This is evident not only from research into the phenomenon of blindsight (Weiskrantz, Barbur, \& Sahraie, 1995; Weiskrantz, Warrington, Sanders, \& Marshall, 1974), but selective behavior in the absence of awareness has also been shown in normal subjects (Boyer, Harrison, \& Ro, 2005; VanRullen \& Koch, 2003) as well as in studies using masked priming (e.g., Vorberg, Mattler, Heinecke, Schmidt, \& Schwarzbach, 2003; Eimer \& Schlaghecken, 1998). Selectivity thus seems an inappropriate indicator of awareness. Therefore, the present experiment employs an objective detection task to assess visibility.

We investigated the effect of pattern masking on the detection of an orientation-defined square in a texture (Figure 1), while recording electroencephalogram (EEG) in human subjects. Figure and no-figure trials were either strongly masked by a pattern mask (masked) leading to chance detection performance, or weakly masked by a uniform gray screen leading to near-perfect detection performance (unmasked). We used a subtraction procedure to control for various confounding influences on the EEG. By subtracting the figure trials from the no-figure trials (Figure 2), two goals were achieved: (1) because both figure and no-figure trials were followed by a mask in the masked condition, any direct influence of the mask on the EEG was subtracted out. The same holds in the unmasked condition. (2) Because both figure and no-figure trials were created using the same set of

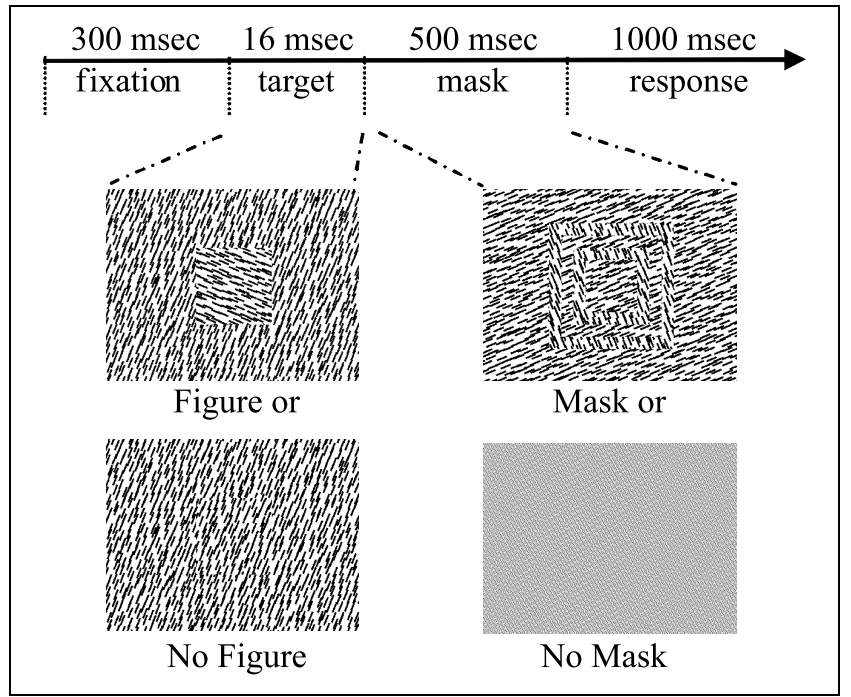

Figure 1. Schematic description of a trial. Subjects were required to indicate whether a figure was present in a texture or not. Half of the trials contained a figure. Half of the trials only contained a homogenous texture. A trial could either be strongly masked (masked) or weakly masked (unmasked). If subjects could not see anything due to masking, they were instructed to guess (forced-choice detection).

oriented line elements, figure and no-figure trials contained exactly equal amounts of contrast and orientation. Thus, any direct influence of low-level processes such as contrast detection and orientation tuning is subtracted out (Zipser, Lamme, \& Schiller, 1996). This subtraction procedure would not work for metacontrast target-mask combinations, as the net result of the subtraction of target-present and target-absent trials would inherently leave a net result of contrast.

The spatio-temporal dynamics of the target-present and target-absent subtraction signal was studied at an extremely short SOA (16.6 msec) where targets were either easily perceived or remained totally undetected as determined by objective measures. We observed marked activation in extrastriate regions in both the visible and invisible condition as a result of feedforward processing. Later and more posterior activation resulting from reentrant processing was observed only in the visible condition. From the results, we infer that reentrant processing is abolished as detection performance drops to chance, even though feedforward processing is entirely preserved.

\section{METHODS}

\section{Participants}

Twenty-one psychology students took part in the experiment in partial fulfillment of first-year course requirements. All subjects (mean age $21.4, \pm 1.9$ ) had normal or corrected-to-normal vision and $71 \%$ were right-handed. Each subject provided written informed consent before 
Figure 2. (A) Examples of stimuli and the schematic orientations scheme. Each gray value represents an orientation; white is isoluminant gray. (B) Representation of subtractions leading to correlates of global processing. Influences of local line elements as well as direct mask contributions are canceled out by ensuring that each orientation is present equally often at each side of the minus sign. Color scheme is the same as in (A).

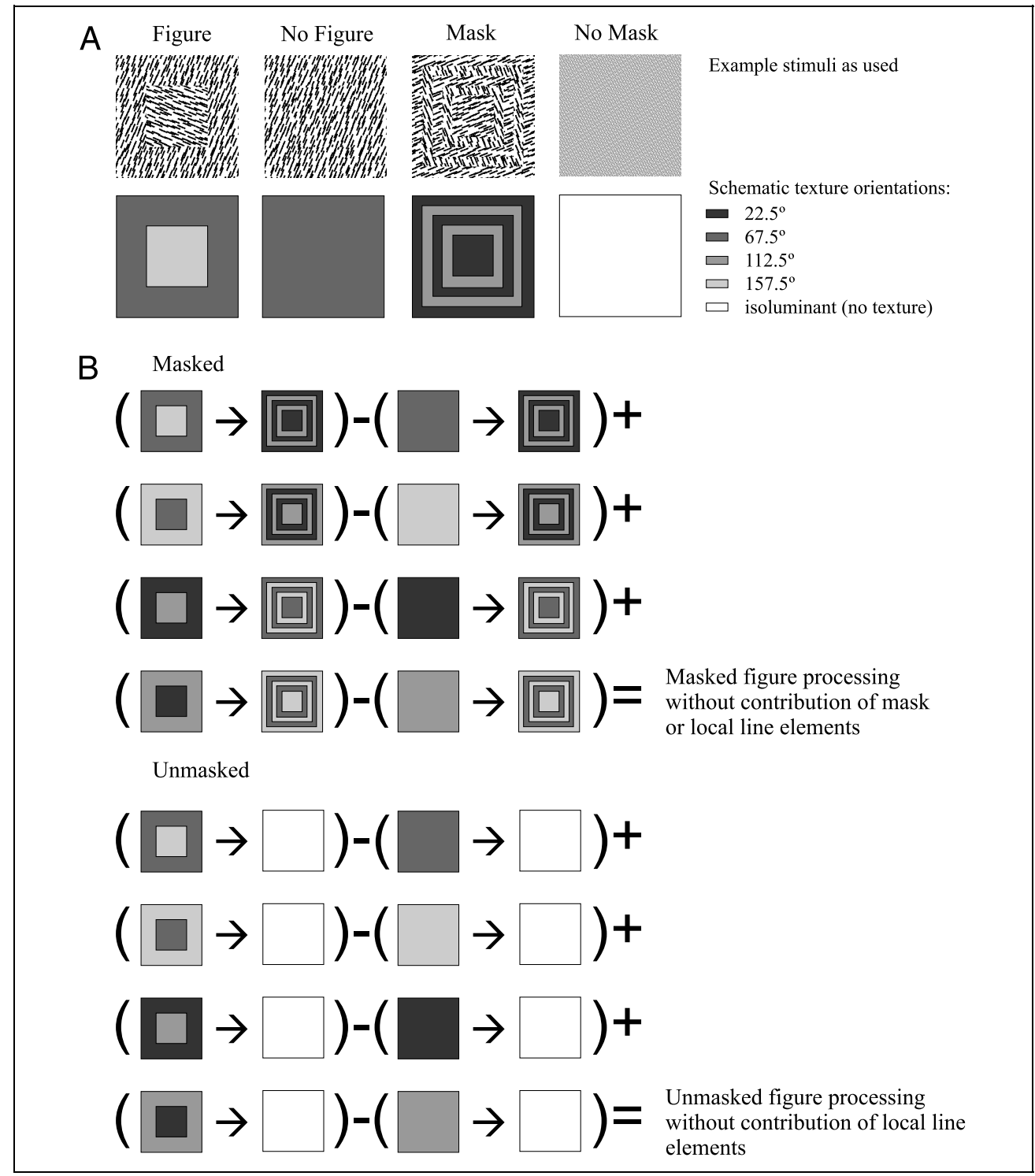

the start of the experiment. All procedures were approved by the ethical committee of the Psychology Department of the University of Amsterdam. Two subjects scored more than two standard deviations below the mean in the unmasked task, indicating difficulty performing the task properly. These were excluded from further analyses. Three subjects scored significantly above chance in the masked condition as ascertained by binomial tests $(p<.05)$, indicating an ability (however slight) to detect the masked figure at this masking interval. These subjects were excluded because it cannot be ruled out that they were still consciously processing these stimuli. In total, five subjects were excluded.

\section{Stimuli and Task}

Without prior training, subjects had to detect the presence or absence of an orientation-defined square in a texture pattern, which was either followed by a mask or by an isoluminant gray screen (all stimuli had a luminance of $72.7 \mathrm{~cd} / \mathrm{m}^{2}$ ). Each of the four resulting trial types was presented an equal number of 300 times in random order throughout the experiment. A trial started with 300 msec fixation, followed by a centrally located target stimulus for $16 \mathrm{msec}$, immediately followed by either a mask or an isoluminant gray screen for $500 \mathrm{msec}$ (Figure 1). Target stimuli and mask consisted of a texture of oriented line elements (approx. $0.07^{\circ}$ thick and $0.4^{\circ}$ long in visual angle). All line elements in a target either had the same orientation (homogenous trials) or contained a centrally located square with line elements orthogonal to the background (figure trials). The square subtended $2.73^{\circ}$ of visual angle. In the masked condition, targets were immediately followed by a texture-defined pattern mask ( $4.54^{\circ}$ visual angle) using orientations not used in the preceding target. In the unmasked condition, targets were followed by an isoluminant gray screen (no texture). A total of four 
orientations of line segments were used for creating figure, homogenous, and mask stimuli $\left(22.5^{\circ}, 67.5^{\circ}, 112.5^{\circ}\right.$, and $157.5^{\circ}$; also see Figure 2 ) so that different orientations for target and mask could always be used within any one trial. All orientations were completely counterbalanced over trials so figure and homogenous trials did not differ from each other with respect to local stimulation (for a similar procedure, see Scholte, Witteveen, Spekreijse, \& Lamme, 2006; Caputo \& Casco, 1999; Lamme, Van Dijk, \& Spekreijse, 1992). Stimuli were created using Matlab (The MathWorks, Inc., Natick, MA, USA). Stimuli were presented using Presentation (Neurobehavioral Systems, Inc., Albany, CA, USA).

Subjects were instructed to fixate throughout the experiment. The fixation dot would turn green $500 \mathrm{msec}$ after target offset to indicate that a response was required, after which the subject had a maximum of $1000 \mathrm{msec}$ to respond before the experiment would continue. On half of the trials, subjects were required to press the left button to indicate square detection and the right button for a homogenous trial. In the other half of the trials, subjects had to switch so that a square was associated with a left button press and a homogenous trial was associated with a right button press. This response scheme was counterbalanced across subjects, half of the subjects starting out square detection with their right hand, and half of the subjects starting out using their left hand. Switching hands posed no difficulty as the high percentages correct on unmasked trials show. Subjects were encouraged to guess if they were unable to see whether a figure was presented, effectively turning the task into a forced-choice task.

\section{Measurements and Analyses}

Event-related potentials (ERPs) were recorded from the scalp using a BioSemi ActiveTwo 48-channel active EEG system (BioSemi, Amsterdam, the Netherlands) at $256 \mathrm{~Hz}$. Forty-eight scalp electrodes were measured (referenced to Fz), as well as two electrodes for horizontal and two for vertical eye movements (each referenced to its opposite counterpart). The data were filtered using a high-pass filter of $1 \mathrm{~Hz}$ and a low-pass filter of $20 \mathrm{~Hz}$, as well as a $50-\mathrm{Hz}$ notch filter. Before ocular correction, automatic artifact rejection was applied by removing segments containing voltage steps of more than $50 \mu \mathrm{V}$, removing any segments falling outside the -200 to $200 \mu \mathrm{V}$ range, and removing those segments containing larger than $300 \mu \mathrm{V}$ differences within the segment. Ocular correction was applied on the basis of the horizontal and vertical electrooculograms (Gratton, Coles, \& Donchin, 1983). After ocular correction, artifact rejection was applied again by removing all segments outside the -75 to $75 \mu \mathrm{V}$ range. Linear local direct current detrending was applied. This is a procedure to remove current drift by subtracting a linear function from each segment. Baseline correction was applied in the $-300 \mathrm{msec}$ to
0 msec interval. All preprocessing steps were conducted using Brain Vision Analyzer (Brain Products, Munich, Germany).

All analyses were conducted on difference waves. These were obtained by subtracting average no-figure trials from figure trials, separately for masked and unmasked trials. Spline Laplacian distribution maps were calculated by interpolating difference waves using spherical splines and approximating current source densities (Perrin, Pernier, Bertrand, \& Echallier, 1989). The resulting maps are spatial second-order derivatives of the scalp potentials lending greater weight to local contributions of cortical generators, filtering out deep sources, as well as being reference free (Nunez \& Srinivasan, 2006).

In order to test at which time points the differences between figure and no-figure trials for the masked and unmasked condition significantly deflect from chance, as well as test the difference between masked and unmasked trials, a random effects analysis was performed by employing a paired two-tailed $t$ test at each time point, treating the average of each subject at that time point as an observation. Correction for multiple comparisons with respect to the number of time points being tested was done by limiting the false discovery rate (FDR), a method in which the $p$ value, at which significance is evaluated, is corrected for the number of tests being performed (Benjamini \& Hochberg, 1995). The method fixes the expected proportion of false positives (the FDR) in contrast to Bonferroni correction, which controls the chance of any false positive among all tests. The FDR is an often used method in a wide variety of scientific fields (for an explanation of how it is used in the field of neuroimaging, see Genovese, Lazar, \& Nichols, 2002). Statistical analyses and visualization of the timecourses were done using Matlab (The MathWorks, Inc.).

\section{RESULTS}

The difference between figure and no-figure trials was easily detected when no mask was present, as expressed in an average detection rate of $96.5 \%( \pm 2.5)$. For the masked trials, the detection rate was at chance at $51.4 \%$ $( \pm 4.1)$. Detection rates are expressed as the average perfect observer score, which is an objective and subject bias-free measure of a subjects' ability to detect a signal (Wickens, 2002). None of the included subjects scored significantly above chance in the masked condition (binomial tests, significance evaluated at .05).

Visual evoked potential (VEP) averages were calculated from the EEG data for each condition. Difference waves of these averages were computed by subtracting no-figure from figure trials in order to isolate activity related to processing of global organization of the target stimulus and discard processing related to processing of local line elements (Figure 2; see also Scholte et al., 
2006; Caputo \& Casco, 1999; Lamme et al., 1992). This was done separately for the masked and unmasked condition, as to subtract out direct contributions of the mask to the VEPs and enable comparison of masked and unmasked trials. All VEP difference waves were converted to spline Laplacian (see Methods) to be able to better isolate local generators of any observed effects and filter out deep sources (Nunez \& Srinivasan, 2006).

A split-half procedure of odd and even trials was used to evaluate the data (Figure 3). Even trials were used for a visualization of instructive time segments using spline Laplacian maps. These were used to select relevant electrodes for pooling. Odd trials were used to evaluate significant deflections of the average current source density at these electrodes over time. Using odd trials for the spatial map and even trials for the timecourse values ensures complete independence of data used for inspection and data used for evaluation of significance. This gives an enormous boost to the reliability of the effects that were found, while precluding coincidental significance of sources that were identified visually. It is important to note that, although for brevity not all time points in the $0-305 \mathrm{msec}$ range have been visualized using scalp distribution maps in Figure 3, electrode pooling was done on the basis of all visually identifiable sources in this domain, whereas none were opportunistically left out. Pooling the data to a limited number of electrodes in this way circumvents the multiple comparisons problem of having to evaluate large numbers of electrodes. Pooling relevant electrodes furthermore increases the signal-to-noise ratio.

Figure $3 \mathrm{~A}$ shows the course of neural processing in the case of unmasked trials. Three stages can be distinguished:

1. A pre-110 msec stage with bilateral anterior occipitotemporal neural generators consistent with feedforward processing toward extrastriate visual areas and beyond (Foxe \& Simpson, 2002).

2. A post-110 msec stage with a clear posterior occipital generator consistent with reentrant processing toward early visual areas, possibly V1.

3. A strong recurring bilateral generator in the 200 $300 \mathrm{msec}$ range, plausibly extrastriate and beyond.

All generators show significant deflections as ascertained by paired $t$ tests between figure and no-figure trials. These were performed on each time point in the time segment, subsequently corrected for multiple comparisons by fixing the FDR at .05 (see Methods).

Figure $3 \mathrm{~B}$ shows the same three stages for the masked trials. Stage 1 again shows a significant bilateral extrastriate neural generator. Stages 2 and 3 are largely absent and show no significant deflections. A direct comparison of the masked and unmasked difference waves was also done (see Figure 4). In accordance with the other results, these showed significant differences in Stage 2 and Stage 3, but not in Stage 1. Taken together, these results are consistent with an interpretation where masking results in an intact initial feedforward activation of extrastriate areas, followed by a disruption of reentrant processing, possibly affecting both striate and extrastriate visual cortex.

Because the resolution of ERPs is rather limited, some may object to the idea that reentrant processing can be pinpointed using ERPs. Yet, in our view, only a relatively simple claim needs to be substantiated: (1) there is early activation found anterior on the scalp; (2) there is distinct later activation posterior on the scalp. In combination with current source densities, and provided that the generators are clearly distinct, this simple anterior/ posterior claim is, in our view, sufficient to claim reentrant processing.

\section{DISCUSSION}

The most salient aspect of these results is that even when subjects are unable to perceive anything by objective measures, widespread bilateral occipito-temporal activation still takes place, showing a clear ability of the brain to pick up differences that the subject cannot express. This early activation is almost indistinguishable from early activity in the visible situation and seems insufficient to generate visual awareness. Some additional process appears necessary in order to achieve conscious perception and detection. The earliest difference between the visible and nonvisible condition arises in the $>110$-msec timeframe, and occurs more posterior than the early bilateral activation. This is most consistent with an interpretation where masking disrupts reentrant processing between high and low visual areas, while leaving feedforward activation intact.

It is unclear how masking accounts resting solely on lateral inhibition could explain these data. The strongest evidence for the influence of lateral inhibition in masking comes from studies employing first-order metacontrast stimuli (e.g., Macknik \& Livingstone, 1998). First-order (luminance-defined) stimuli are obvious candidates for spatio-temporal lateral inhibitory influences, as one can see how a metacontrasting stimulus laterally inhibits a first-order contrast stimulus of opposite polarity. However, the present study makes use of secondorder target stimuli and pattern masking. As target and mask do not bear a metacontrasting relation to each other, low-level lateral inhibitory influences are less likely. Moreover, any remains of low-level inhibitory influences that may still exist are subtracted out using the figure minus no-figure subtraction.

However, one could still postulate that high-level lateral inhibitory mechanisms operate between complex representations-as suggested by Macknik and MartinezConde (2004). If this were the case, one would predict the pre-110 msec activation to be disrupted in the masked scenario as a result of these inhibitory influ- 


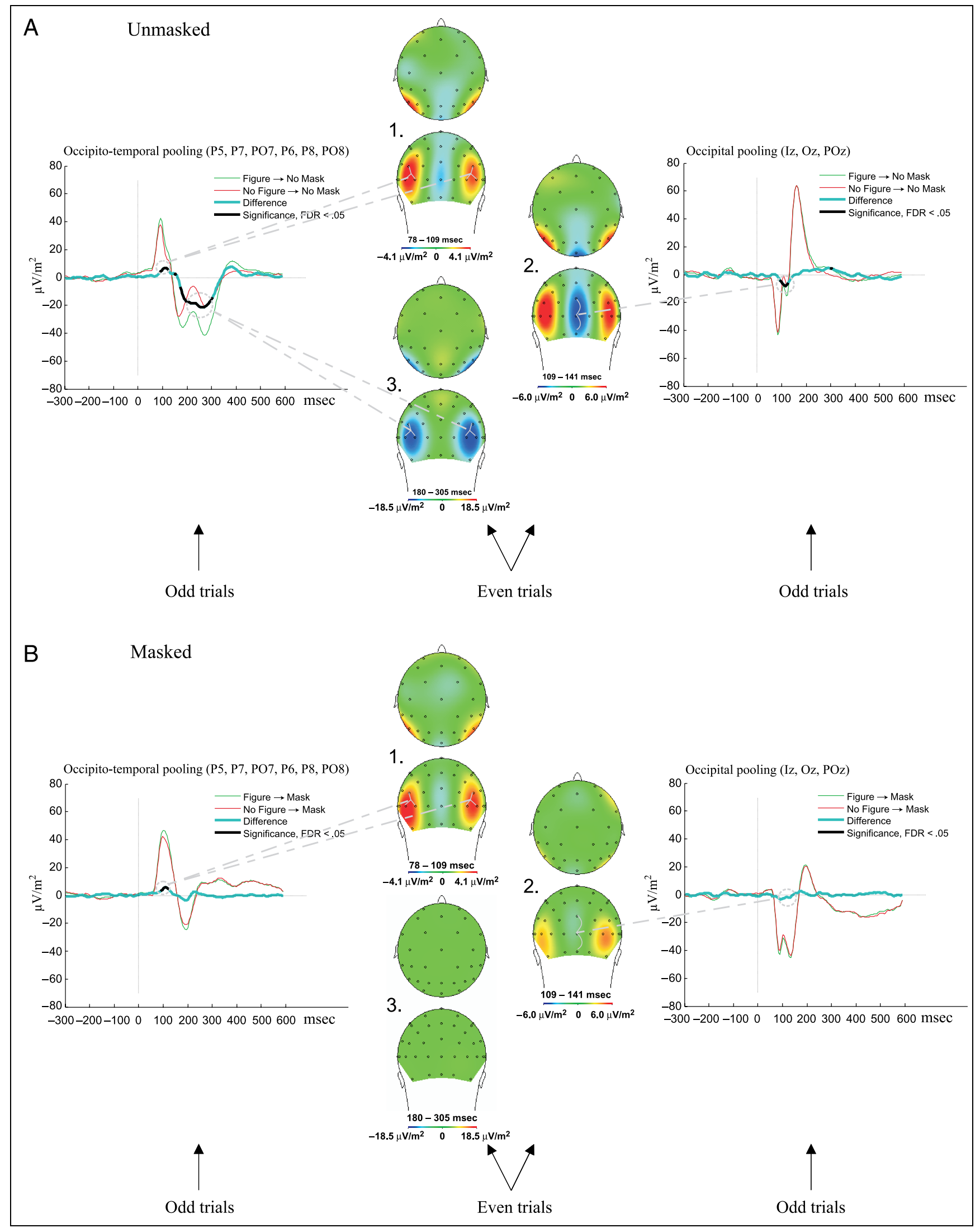

Figure 3. Results. Spline Laplacian maps over three time segments: (1) 78-109 msec, (2) 109-141 msec, and (3) 180-305 msec, and event-related averages of relevant electrodes (pooled occipitally and occipito-temporally). Even trials were used for data inspection (the maps) to determine sensible electrode poolings. Odd trials were used for the averages and evaluation of statistical significance. (A) Unmasked condition; (B) Masked condition. Both masked and unmasked show strong significant temporal bilateral activation reflecting feedforward activation in Stage 1. More posterior occipital activation reflecting reentrant processing can only be seen in the unmasked condition in Stage 2, but is abolished by masking. 


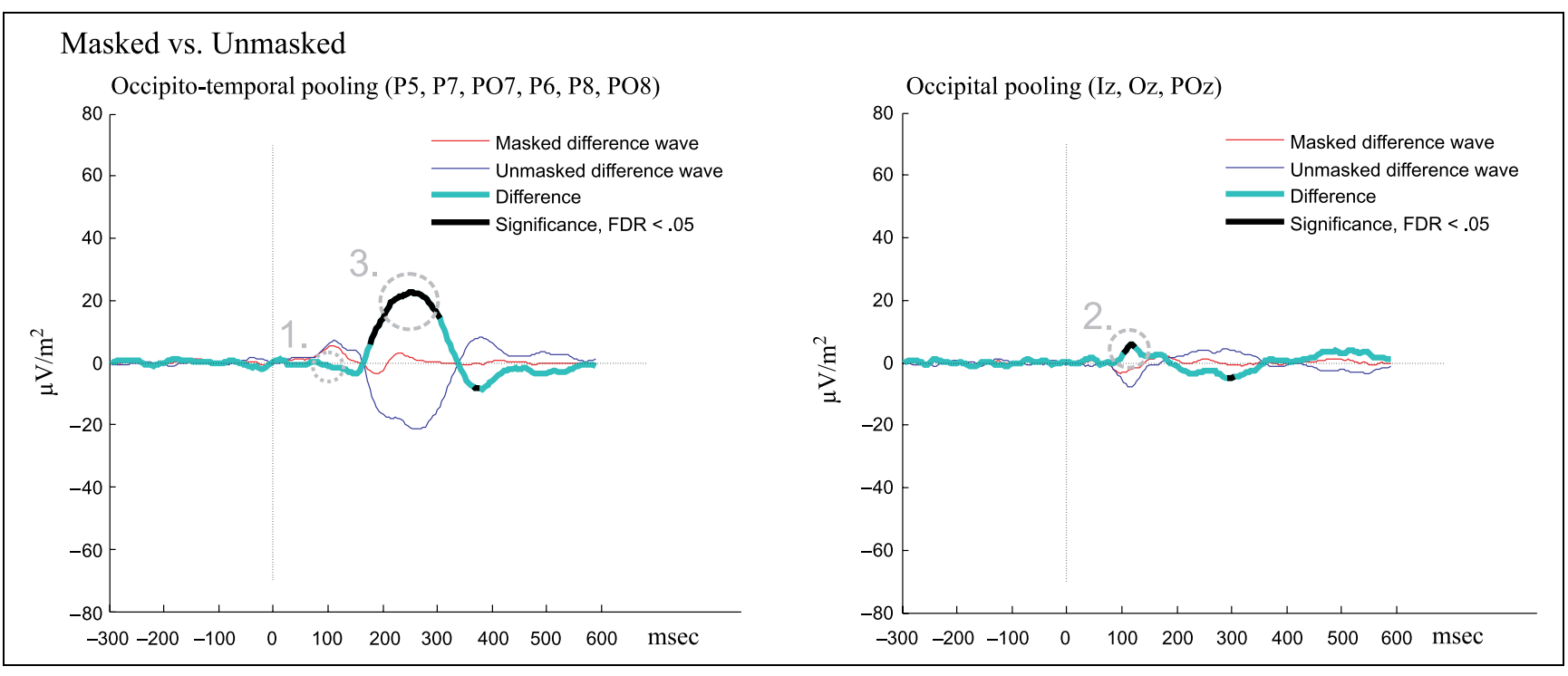

Figure 4. A difference wave of the difference waves outlined in Figure 3 was calculated so as to directly test at which intervals the masked and the unmasked figures differ from each other. This figure clearly shows that masked and unmasked trials do not significantly differ from each other in the early 78-109 msec occipito-temporal stage related to feedforward processing (1), but do differ significantly from each other in the 109-141 msec occipital stage (2), which is plausibly related to reentrant processing as well as the later 180-305 msec occipito-temporal stage (3).

ences. However, the locus of interruption in this experiment is later in time and more posterior. This is not to say that lateral inhibitory influences do not play a role in many types of masking, only that the view of ubiquitous lateral inhibition as an explanation for our results is not tenable.

Interchannel inhibition accounts of masking presume the existence of two channels in visual processing, a fast and a slow one. Masking is presumably caused by the mask in a fast-acting channel inhibited by the processing of the target in a slow-acting channel. Such accounts cannot be ruled out entirely by these data, as such theories do not specify at which level inhibition takes place. It could be argued that the feedforward activity found in this study is caused by target activity in a fastacting channel, and that the posterior $>110$-msec activity, which we call reentrant activity, is caused by a slow-acting channel. In the masked scenario, this activity would be inhibited by the mask through subsequent activation of the fast-acting channel (which would have to be projecting downward to inhibit the slow-acting channel). However, aside from the fact that the posterior activity we observed is not very "sustained" as such models propose, there is little neurophysiological evidence that interchannel inhibition actually exists (also see Enns \& Di Lollo, 2000). This makes an explanation built on proven notions of reentrant processing much more parsimonious in this context.

Aside from the present study, a number of other studies have inferred the influence of masking on reentrant processing in human subjects, most notably studies employing object substitution masking (Weidner, Shah, \& Fink, 2006; Woodman \& Luck, 2003; Di Lollo et al., 2000). In object substitution masking (also referred to as four-dot masking), a four-dot mask is presented surrounding a nonattended target. The subsequent discrimination of this target is unimpaired when target and mask coterminate, but strongly impaired when the dots remain visible after target offset. This is explained by postulating that the four dots (after offset of the target) create a mismatch between the cortical representation of the four dots in early visual areas and the cortical representation of the target in higher areas. Although we believe that the results of these studies have significant implications for the influence of reentrant processing on perception, they differ from our results in important ways.

Four-dot masking depends on attention being directed elsewhere and increases with increasing set size (Enns, 2004; Di Lollo et al., 2000). In our pattern masking study, attention is fully directed on the target, and set size is just one. Moreover, uninterrupted processing of masked targets using object substitution continues well into the 200-300 msec domain (Woodman \& Luck, 2003), whereas our results show that processing is disrupted at $110 \mathrm{msec}$. Indeed, a recent fMRI study comparing pattern and object substitution masking shows that differences between the two can be found in the left middle frontal gyrus, in the precentral gyrus of the right hemisphere, and in the medial superior frontal gyrus (Weidner et al., 2006), whereas our study shows strictly occipital disruption due to pattern masking.

What could account for these differences? What happens during object substitution masking seems to be more akin to what happens during other attentional manipulations, such as the attentional blink. Many attentional blink studies have shown that processing of unseen target stimuli goes almost completely uninterrupted up to $300 \mathrm{msec}$ and that differences between 
reported and unreported targets are to be found in frontal and parietal cortices (Kranczioch, Debener, Schwarzbach, Goebel, \& Engel, 2005; Sergent, Baillet, \& Dehaene, 2005; Luck, Vogel, \& Shapiro, 1996). This is reminiscent of the response profile found in object substitution masking. As other authors have noted, the failure of subjects to report stimuli in such instances may reflect a loss of information at a postperceptual stage, where information is replaced before it can be transferred to working memory and accessible awareness (e.g., Woodman \& Luck, 2003; Luck et al., 1996).

It may still be reentrant processing that is interrupted, but at a later stage and between visual and higher brain areas (possibly frontal and parietal), whereas early reentrant processing within visual areas goes uninterrupted. Recent models of consciousness proposed by Dehaene, Changeux, Naccache, Sackur, and Sergent (2006) and Lamme $(2003,2006)$ outline similar ideas in more detail. The notion of several reentrant or recurrent loops at multiple levels is highly likely given the temporal dynamics of cortical processing (e.g., Foxe \& Simpson, 2002). Because of the +110 -msec timing and location observed in this experiment, we conclude that reentrant processing was disrupted at perceptual levels early on, comparable to what has been shown in other studies using different stimuli (Haynes, Driver, \& Rees, 2005; Breitmeyer et al., 2004; Ro et al., 2003).

The function of reentrant processing within occipital cortex is a somewhat open question, although strong evidence exists that it is of importance for figure-ground segmentation (e.g., Lamme, Rodriguez-Rodriguez, \& Spekreijse, 1999; Hupe et al., 1998; Lamme, 1995). In such studies, it is typically shown that a neuron in V1 starts responding differentially after about $100 \mathrm{msec}$ when its classical receptive field (CRF) is located on the center of a figure compared to when it is on a background, despite local stimulation being exactly equal (cf. Figure 2). These extra-CRF effects are usually referred to as contextual modulation, as they are modulated by context information outside their CRF. The presence and timing $(>100 \mathrm{msec}$ ) of contextual modulation strongly suggests that reentrant neural processing takes place going back from higher areas containing larger receptive fields to lower areas such as V1. Also, when extrastriate regions are lesioned, contextual modulation disappears, leaving receptive field tuning properties intact and conclusively showing the role of reentrant processing in contextual modulation (Lamme, Super, \& Spekreijse, 1998).

A study in which the effect of target-mask SOA on contextual modulation in macaque V1 was investigated showed a strong relation between SOA, contextual modulation, and the monkey's ability to detect a texturedefined figure (Lamme et al., 2002). Moreover, any influence of SOA on the CRF tuning properties of these neurons was almost completely absent, showing that feedforward activation was largely preserved. However, because measurements were done only in V1, it was unclear to what extent feedforward activation would continue to higher areas and to what extent the same would hold for humans.

The present study clearly demonstrates that in humans, undetected and strongly masked texture stimuli are processed far beyond the striate cortex (see Figure 3), showing that feedforward processing is preserved, whereas reentrant processing is selectively interrupted. A neural network model of figure-ground segmentation by Roelfsema, Lamme, Spekreijse, and Bosch (2002), in which texture segregation in macaques is modeled in terms of feedforward and reentrant processing, serves extremely well in explaining the spatio-temporal profile of such results.

Related models propose that reentrant processing is more intimately related to visual awareness (e.g., Ro et al., 2003; Lamme, 2001). These views on reentrant processing are not necessarily contradicting, as visual awareness might simply be the way surface segregation and related processes express themselves phenomenologically. The view that reentrant activity in the visual cortex correlates with visual awareness is now supported by converging evidence from monkey physiology (e.g., Lamme, Super, Landman, Roelfsema, \& Spekreijse, 2000), EEG (the present study), transcranial magnetic stimulation (Pascual-Leone \& Walsh, 2001), and fMRI (e.g., Haynes et al., 2005).

\section{Conclusion}

We argue and found evidence for at least three stages in early visual processing: (1) a completely unconscious pre-110 msec feedforward stage, which is probably involved in boundary detection but goes uninterrupted by masking; (2) a second stage, which operates in the $110-140$ msec range reflecting reentrant processing in visual cortex, plausibly encapsulating processes such as figure-ground segmentation and phenomenal awareness; and (3) a stage in the 200-300 msec range, which is contingent upon earlier stages. Specifically, the second stage was interrupted by backward masking, confirming that pre-110 msec activation proceeds outside awareness, whereas more posterior $>110$ msec activity is (at least) a necessary condition for awareness to arise. Finally, we reject explanations of backward masking as a unitary phenomenon, in agreement with other authors such as Bachmann, Luiga, and Poder (2005). Any explanation of the masking phenomenon should carefully take into account physical stimulus characteristics and how these are processed by the various components of the visual system.

\section{Acknowledgments}

We thank IJsbrand Attema for his help in collecting the data for this experiment, as well as the anonymous reviewers for their helpful comments. 
Reprint requests should be sent to J. J. Fahrenfort, Department of Psychology, University of Amsterdam, Roetersstraat 15, 1018 WB Amsterdam, the Netherlands, or via e-mail: j.j.fahrenfort@ uva.nl.

\section{REFERENCES}

Bachmann, T., Luiga, I., \& Poder, E. (2005). Variations in backward masking with different masking stimuli: II. The effects of spatially quantised masks in the light of local contour interaction, interchannel inhibition, perceptual retouch, and substitution theories. Perception, 34, 139-153.

Becker, M. W., \& Anstis, S. (2004). Metacontrast masking is specific to luminance polarity. Vision Research, 44 , $2537-2543$.

Benjamini, Y., \& Hochberg, Y. (1995). Controlling the false discovery rate-A practical and powerful approach to multiple testing. Journal of the Royal Statistical Society, Series B, Methodological, 57, 289-300.

Boyer, J. L., Harrison, S., \& Ro, T. (2005). Unconscious processing of orientation and color without primary visual cortex. Proceedings of the National Academy of Sciences, U.S.A., 102, 16875-16879.

Breitmeyer, B. G., Ro, T., \& Ogmen, H. (2004). A comparison of masking by visual and transcranial magnetic stimulation: Implications for the study of conscious and unconscious visual processing. Consciousness and Cognition, 13, 829-843.

Bridgeman, B. (1980). Temporal response characteristics of cells in monkey striate cortex measured with metacontrast masking and brightness discrimination. Brain Research, 196, 347-364.

Caputo, G., \& Casco, C. (1999). A visual evoked potential correlate of global figure-ground segmentation. Vision Research, 39, 1597-1610.

Dehaene, S., Changeux, J. P., Naccache, L., Sackur, J., \& Sergent, C. (2006). Conscious, preconscious, and subliminal processing: A testable taxonomy. Trends in Cognitive Sciences, 10, 204-211.

Dehaene, S., Naccache, L., Cohen, L., Le Bihan, D., Mangin, J. F., Poline, J. B., et al. (2001). Cerebral mechanisms of word masking and unconscious repetition priming. Nature Neuroscience, 4, 752-758.

Dehaene, S., Naccache, L., Le Clec'H, G., Koechlin, E., Mueller, M., Dehaene Lambertz, G., et al. (1998). Imaging unconscious semantic priming. Nature, 395, 597-600.

Di Lollo, V., Enns, J. T., \& Rensink, R. A. (2000). Competition for consciousness among visual events: The psychophysics of reentrant visual processes. Journal of Experimental Psychology: General, 129, 481-507.

Eimer, M., \& Schlaghecken, F. (1998). Effects of masked stimuli on motor activation: Behavioral and electrophysiological evidence. Journal of Experimental Psychology: Human Perception and Performance, 24, 1737-1747.

Enns, J. T. (2004). Object substitution and its relation to other forms of visual masking. Vision Research, 44 , $1321-1331$.

Enns, J. T., \& Di Lollo, V. (2000). What's new in visual masking? Trends in Cognitive Sciences, 4, 345-352.

Felleman, D. J., \& Van Essen, D. C. (1991). Distributed hierarchical processing in the primate cerebral cortex. Cerebral Cortex, 1, 1-47.

Foxe, J. J., \& Simpson, G. V. (2002). Flow of activation from V1 to frontal cortex in humans-A framework for defining "early" visual processing. Experimental Brain Research, 142, 139-150.
Francis, G. (1997). Cortical dynamics of lateral inhibition: Metacontrast masking. Psychological Review, 104, 572-594.

Genovese, C. R., Lazar, N. A., \& Nichols, T. (2002). Thresholding of statistical maps in functional neuroimaging using the false discovery rate. Neuroimage, 15, 870-878.

Gratton, G., Coles, M. G., \& Donchin, E. (1983). A new method for off-line removal of ocular artifact. Electroencephalography and Clinical Neurophysiology, 55, 468-484

Greenwald, A. G., Klinger, M. R., \& Liu, T. J. (1989). Unconscious processing of dichoptically masked words. Memory \& Cognition, 17, 35-47.

Hartline, H. K. (1949). Inhibition of activity of visual receptors by illuminating nearby retinal areas in the Limulus eye. Federation Proceedings, 8, 69.

Haynes, J. D., Driver, J., \& Rees, G. (2005). Visibility reflects dynamic changes of effective connectivity between V1 and fusiform cortex. Neuron, 46, 811-821.

Hochstein, S., \& Ahissar, M. (2002). View from the top: Hierarchies and reverse hierarchies in the visual system. Neuron, 36, 791-804.

Hupe, J. M., James, A. C., Payne, B. R., Lomber, S. G., Girard, P., \& Bullier, J. (1998). Cortical feedback improves discrimination between figure and background by V1, V2 and V3 neurons. Nature, 394, 784-787.

Kastner, S., \& Ungerleider, L. G. (2000). Mechanisms of visual attention in the human cortex. Annual Review of Neuroscience, 23, 315-341.

Kovacs, G., Vogels, R., \& Orban, G. A. (1995). Cortical correlate of pattern backward-masking. Proceedings of the National Academy of Sciences, U.S.A., 92, 5587-5591.

Kranczioch, C., Debener, S., Schwarzbach, J., Goebel, R., \& Engel, A. K. (2005). Neural correlates of conscious perception in the attentional blink. Neuroimage, 24, 704-714.

Lamme, V. A. F. (1995). The neurophysiology of figure ground segregation in primary visual-cortex. Journal of Neuroscience, 15, 1605-1615.

Lamme, V. A. F. (2001). Blindsight: The role of feedforward and feedback corticocortical connections. Acta Psychologica, 107, 209-228.

Lamme, V. A. F. (2003). Why visual attention and awareness are different. Trends in Cognitive Sciences, 7, 12-18.

Lamme, V. A. F. (2006). Towards a true neural stance on consciousness. Trends in Cognitive Sciences, 10, 494-501.

Lamme, V. A. F., Rodriguez-Rodriguez, V., \& Spekreijse, H. (1999). Separate processing dynamics for texture elements, boundaries and surfaces in primary visual cortex of the macaque monkey. Cerebral Cortex, 9, 406-413.

Lamme, V. A. F., \& Roelfsema, P. R. (2000). The distinct modes of vision offered by feedforward and recurrent processing. Trends in Neurosciences, 23, 571-579.

Lamme, V. A. F., Super, H., Landman, R., Roelfsema, P. R., \& Spekreijse, H. (2000). The role of primary visual cortex (V1) in visual awareness. Vision Research, 40, 1507-1521.

Lamme, V. A. F., Super, H., \& Spekreijse, H. (1998). Feedforward, horizontal, and feedback processing in the visual cortex. Current Opinion in Neurobiology, 8, 529-535.

Lamme, V. A. F., Van Dijk, B. W., \& Spekreijse, H. (1992). Texture segregation is processed by primary visual cortex in man and monkey. Evidence from VEP experiments. Vision Research, 32, 797-807.

Lamme, V. A. F., Zipser, K., \& Spekreijse, H. (2002). Masking interrupts figure-ground signals in V1. Journal of Cognitive Neuroscience, 14, 1044-1053. 
Luck, S. J., Chelazzi, L., Hillyard, S. A., \& Desimone, R. (1997). Neural mechanisms of spatial selective attention in areas V1, V2, and V4 of macaque visual cortex. Journal of Neurophysiology, 77, 24-42.

Luck, S. J., Vogel, E. K., \& Shapiro, K. L. (1996). Word meanings can be accessed but not reported during the attentional blink. Nature, 383, 616-618.

Macknik, S. L., \& Livingstone, M. S. (1998). Neuronal correlates of visibility and invisibility in the primate visual system. Nature Neuroscience, 1, 144-149.

Macknik, S. L., \& Martinez-Conde, S. (2004). Dichoptic visual masking reveals that early binocular neurons exhibit weak interocular suppression: Implications for binocular vision and visual awareness. Journal of Cognitive Neuroscience, 16, 1049-1059.

Naccache, L., Gaillard, R., Adam, C., Hasboun, D., Clemenceau, S., Baulac, M., et al. (2005). A direct intracranial record of emotions evoked by subliminal words. Proceedings of the National Academy of Sciences, U.S.A., 102, 7713-7717.

Nunez, P., \& Srinivasan, R. (2006). Electric fields of the brain. Oxford: Oxford University Press.

Ogmen, H., Breitmeyer, B. G., \& Melvin, R. (2003). The what and where in visual masking. Vision Research, 43, $1337-1350$

Pascual-Leone, A., \& Walsh, V. (2001). Fast backprojections from the motion to the primary visual area necessary for visual awareness. Science, 292, 510-512.

Perrin, F., Pernier, J., Bertrand, O., \& Echallier, J. F. (1989). Spherical splines for scalp potential and current-density mapping. Electroencephalography and Clinical Neurophysiology, 72, 184-187.

Rao, R. P. N., \& Ballard, D. H. (1999). Predictive coding in the visual cortex: A functional interpretation of some extra-classical receptive-field effects. Nature Neuroscience, 2, 79-87.

Ro, T., Breitmeyer, B., Burton, P., Singhal, N. S., \& Lane, D. (2003). Feedback contributions to visual awareness in human occipital cortex. Current Biology, 13, 1038-1041.

Roelfsema, P. R., Lamme, V. A. F., Spekreijse, H., \& Bosch, H. (2002). Figure-ground segregation in a recurrent network architecture. Journal of Cognitive Neuroscience, 14, $525-537$.
Rolls, E. T., \& Tovee, M. J. (1994). Processing speed in the cerebral-cortex and the neurophysiology of visual masking. Proceedings of the Royal Society of London, Series B, Biological Sciences, 257, 9-15.

Scholte, H. S., Witteveen, S. C., Spekreijse, H., \& Lamme, V. A. F. (2006). The influence of inattention on the neural correlates of scene segmentation. Brain Research, 1076, 106-115.

Sergent, C., Baillet, S., \& Dehaene, S. (2005). Timing of the brain events underlying access to consciousness during the attentional blink. Nature Neuroscience, 8, 1391-1400.

Spratling, M. W., \& Johnson, M. H. (2004). A feedback model of visual attention. Journal of Cognitive Neuroscience, 16, 219-237.

Thompson, K. G., \& Schall, J. D. (1999). The detection of visual signals by macaque frontal eye field during masking. Nature Neuroscience, 2, 283-288.

VanRullen, R., \& Koch, C. (2003). Visual selective behavior can be triggered by a feed-forward process. Journal of Cognitive Neuroscience, 15, 209-217.

Vorberg, D., Mattler, U., Heinecke, A., Schmidt, T., \& Schwarzbach, J. (2003). Different time courses for visual perception and action priming. Proceedings of the National Academy of Sciences, U.S.A., 100, 6275-6280.

Weidner, R., Shah, N. J., \& Fink, G. R. (2006). The neural basis of perceptual hypothesis generation and testing. Journal of Cognitive Neuroscience, 18, 258-266.

Weiskrantz, L., Barbur, J. L., \& Sahraie, A. (1995). Parameters affecting conscious versus unconscious visual-discrimination with damage to the visual-cortex (V1). Proceedings of the National Academy of Sciences, U.S.A., 92, 6122-6126.

Weiskrantz, L., Warrington, E. K., Sanders, M. D., \& Marshall, J. (1974). Visual capacity in the hemianopic field following a restricted occipital ablation. Brain, 97, 709-728.

Wickens, T. D. (2002). Elementary signal detection theory. Oxford: Oxford University Press.

Woodman, G. F., \& Luck, S. J. (2003). Dissociations among attention, perception, and awareness during object-substitution masking. Psychological Science, 14, 605-611.

Zipser, K., Lamme, V. A. F., \& Schiller, P. H. (1996). Contextual modulation in primary visual cortex. Journal of Neuroscience, 16, 7376-7389. 